\title{
Uso de fatores de crescimento epidérmico e estimulador de colônias de granulócitos na prevenção e tratamento da enterocolite necrosante no recém-nascido
}

\author{
Use of epidermic and granulocyte-colony stimulating growth factors in the prevention and treatment of \\ necrotizing enterocolitis of the newborn
}

\begin{abstract}
Dáfne Cardoso B. da Silva ${ }^{1}$, Camila Quinello ${ }^{1}$, Denise Amazonas Pires ${ }^{1}$, Juliana Rodrigues Pinto ${ }^{1}$, Ana Cláudia Mattar ${ }^{1}$, Vera Lucia J. Krebs², Maria Esther J. R. Ceccon²
\end{abstract}

\section{RESUMO}

Objetivo: Revisar os conhecimentos existentes em relação ao uso de fatores de crescimento epidérmico e estimulador de colônias de granulócitos na prevenção e/ou no tratamento da enterocolite necrosante (ECN) durante o período neonatal.

Fontes de dados: Revisão da literatura, nas bases de dados Medline, Lilacs, SciELO e PubMed, utilizando os unitermos "recém-nascidos", "enterocolite" e "fatores de crescimento", no período de 2003 a 2007. Nesta busca, 49 artigos foram encontrados, sendo 17 pertinentes ao tema. Também foram utilizados outros artigos, independente do ano de publicação, relacionados a aspectos definidores da ECN no recém-nascido.

Síntese dos dados: A ECN continua sendo responsável por uma elevada morbimortalidade neonatal. Os mecanismos fisiopatológicos vêm sendo elucidados e, a partir deles, são discutidas novas terapias, como o uso de fatores de crescimento, destacando-se o fator de crescimento epidérmico e o fator estimulador de colônias de granulócitos.

Conclusões: $\mathrm{O}$ uso de fatores de crescimento no tratamento e prevenção da ECN neonatal parece promissor. É necessário maior número de ensaios clínicos para comprovar sua eficácia e segurança. Enquanto isso, a melhor prática médica continua sendo a prevenção da doença.

Palavras-chave: recém-nascido; dietoterapia; fatores de crescimento; enterocolite necrosante.

\section{ABSTRACT}

Objective: To review the literature regarding the use of hematopoietic and epidermic growth factors for prevention or treatment of neonatal necrotizing enterocolitis (NEC).

Data sources: Literature review of Medline, Lilacs, SciELO and Pubmed databases, using the key-words "newborn", "enterocolitis" and "growth factors", from 2003 to 2007. Fourty-nine papers were retrieved, but only 17 related to the subject. Other studies that described some clinical aspects of enterocolitis were also included, regardless of the year of publication.

Data synthesis: Necrotizing and enterocolitis has been an important cause of morbidity and mortality in the neonatal period. As the knowledge about the pathophysiology of this disease improves, new therapies, such as the administration of epidermal growth factor and granulocyte colony-stimulating factor, are being discussed.

Conclusions: The use of growth factors for treatment and prevention of NEC seems promising. However, further clinics assays are needed to evaluate the effectiveness and the safety of these growth factors. At this moment, the best clinical practice is the prevention of the disease.

Key-words: infant, newborn; diet therapy; growth factors; enterocolitis, necrotizing.
${ }^{1}$ Aluna do curso de Pós-graduação Senso Estrito do Departamento de Pediatria da Faculdade de Medicina da Universidade de São Paulo (FMUSP), São Paulo, SP, Brasil

2Livre-docente em Pediatria pela FMUSP e orientadora do curso de Pósgraduação Senso Estrito do Departamento de Pediatria da FMUSP, São Paulo, SP, Brasil
Endereço para correspondência:

Maria Esther J. R. Ceccon

Rua Apiacás, 570, apto. 104 - Perdizes

CEP 05017-020

E-mail: mariaejc@icr.hcnet.usp.br

Recebido em: 1/11/2007

Aprovado em: 24/2/2008 


\section{Introdução}

A enterocolite necrosante (ECN) é a doença gastrintestinal mais comum nos recém-nascidos $(\mathrm{RN})$ internados nas unidades de terapia intensiva, sendo responsável por elevada morbidade e mortalidade, principalmente em prematuros com peso de nascimento inferior a $1.500 \mathrm{~g}^{(1-3)}$

Sua etiologia parece multifatorial, sendo considerados como principais fatores de risco a prematuridade, os episódios de diminuição da perfusão do intestino (isquemia transitória), a composição da dieta administrada ao $\mathrm{RN}$ e a velocidade de progressão da alimentação enteral, além da colonização bacteriana ${ }^{(4,5)}$.

No início do quadro clínico, o $\mathrm{RN}$ apresenta distensão de alças intestinais, edema, aumento do volume de líquido peritonial e hematoquezia. A camada serosa do intestino mostra-se edemaciada e recoberta por fibrina. Pode ocorrer necrose da mucosa em alguns segmentos do intestino que, com o progredir da doença, passa a comprometer as demais camadas da parede e maiores extensões do intestino. O segmento mais acometido é o íleo terminal, seguido pelo cólon e pelo jejuno. Pode também haver sinais de peritonite, sepse, choque séptico e insuficiência de múltiplos órgãos. Os microrganismos mais comumente isolados na ECN são a Klebsiella sp e o Staphylococcus s $p^{(3)}$

A característica radiológica típica da ECN é a pneumatose intestinal e a distensão de alças intestinais; em alguns casos, observa-se pneumoperitôneo e gás no sistema porta. Este gás é o hidrogênio resultante do metabolismo bacteriano ${ }^{(3)}$. Bell et al, em 1978, estabeleceram critérios para classificar os estágios da ECN, adaptados em 1986 e em vigor até os dias atuais (Quadro 1$)^{(6,7)}$.

Quando a doença evolui para perfuração intestinal, o paciente é, em geral, submetido à laparotomia exploradora, com ressecção do segmento acometido. Na evolução, pode haver estenose deste segmento e/ou síndrome do intestino curto. Esta morbidade responde por comprometimento da qualidade de vida do paciente e por elevado custo de assistência à saúde. Apesar dos progressos alcançados na terapia intensiva neonatal, a mortalidade pela ECN não apresentou redução nos últimos anos e situa-se entre 18 e $45 \%$, dependendo do grau de prematuridade e da gravidade da doença ${ }^{(4,8)}$.

Devido às elevadas taxas de morbidade e mortalidade, vêm sendo estudadas novas estratégias de prevenção e tratamento da ECN, focadas nos possíveis mecanismos fisiopatológicos, considerando que a imaturidade da motilidade, absorção e defesa gastrintestinais contribua para o alto risco e aumento da suscetibilidade das crianças prematuras à ECN.

Devido à associação entre ECN e uso de fórmula na nutrição dos prematuros, a composição biologicamente ativa do leite materno passou a ser objeto de estudo ${ }^{(9-11)}$. Um grande número de peptídeos biologicamente ativos foi identificado em concentrações significativas no colostro e leite humano; dentre eles, o fator de crescimento epidérmico (EGF), o fator estimulador de colônias de granulócitos (G-CSF) e a eritropoetina $(\mathrm{EPO})^{(12)}$. Além disso, o líquido amniótico deglutido pelo feto no último trimestre de gestação também contém concentrações significativas de fatores de crescimento, que gradualmente aumentam durante a gestação, alcançando maiores níveis ao final da gestação a termo ${ }^{(8-10,13-15)}$.

$\mathrm{O}$ uso de fatores de crescimento para o tratamento de doenças gastrintestinais é associado com preocupações sobre seus riscos potenciais. Fatores de crescimento administrados sistemicamente poderiam induzir a proliferação celular em outras regiões do corpo, com risco teórico de malignidade. Portanto, a administração destes fatores de crescimento deveria ser feita diretamente no local da lesão intestinal, de forma enteral.

Neste contexto, o objetivo da presente revisão foi sistematizar o conhecimento atual acerca do uso do EGF e do G-CSF na prevenção e/ou tratamento da ECN.

\section{Fator estimulador de colônias de granulócitos}

Um dos fatores de crescimento hematopoético, o G-CSF, possui ação já bem estabelecida nas linhagens de células sanguíneas. Entretanto, seus receptores também podem ser encontrados em abundância nos enterócitos. O G-CSF pode

Quadro 1 - Critérios de Bell modificados

\begin{tabular}{|llll|}
\hline Estágios & Classificação & Sinais clínicos & Sinais radiológicos \\
\hline II & Suspeita & $\begin{array}{l}\text { Distensão abdominal, resíduo gástrico, fezes } \\
\text { com sangue, apnéia, letargia }\end{array}$ & Dilatação de alças, íleo paralítico \\
III & Comprovada & $\begin{array}{l}\text { I + abdome dolorido, acidose metabólica, } \\
\text { plaquetopenia }\end{array}$ & $\begin{array}{l}\text { Pneumatose intestinal, gás no } \\
\text { sistema porta }\end{array}$ \\
& Avançada & $\begin{array}{l}\text { II + hipotensão, acidose importante, coagulação } \\
\text { intravascular dissiminada, neutropenia }\end{array}$ & Estágio II + pneumoperitônio \\
\hline
\end{tabular}


estar ligado ao desenvolvimento do intestino, porém, o mecanismo pelo qual isto ocorre ainda não é bem conhecido.

Estudos em animais demonstraram que o G-CSF administrado por via oral não foi capaz de alterar a contagem de neutrófilos absoluta ou relativa, mas teve efeitos na área da vilosidade intestinal, no comprimento, no perímetro e na profundidade das criptas intestinais e na expressão do antígeno marcador da multiplicação das células da cripta.

Calhoun et al estudaram $20 \mathrm{RN}$ prematuros e de termo, em ensaio clínico prospectivo, randomizado e duplo-cego. Foi comparada a absorção de $100 \mu \mathrm{g} / \mathrm{kg}$ de rhG-CSF, administrado via enteral, versus placebo, com 48 horas de intervalo entre as duas dietas. $\mathrm{O}$ estudo concluiu que o rhG-CSF não era absorvido em quantidades significativas, já que os níveis plasmáticos de G-CSF dos grupos não apresentaram diferença estatística $(\mathrm{p}=0,68$, quando comparados de acordo com a idade gestacional). Os autores sugeriram que o efeito do G-CSF por via enteral era local e não sistêmico ${ }^{(12,16)}$.

Canpolat et al, em 2006, publicaram um ensaio clínico com prematuros com enterocolite grau I, randomizados para receber placebo $(n=10)$ ou rhG-CSF por via enteral $(n=8)$, $20 \mu \mathrm{g} / \mathrm{kg} /$ dia durante cinco dias, a partir do primeiro dia do diagnóstico. Nenhum dos prematuros do grupo rhG-CSF, comparados a $50 \%$ do grupo controle, apresentou progressão da doença para estágios II ou III $(p<0,05)$. Neste trabalho, o rhG-CSF não apenas se mostrou capaz de impedir a progressão da enterocolite, como também diminuiu o tempo para a resolução da doença $(p<0,001)^{(17)}$.

\section{Fator de crescimento epidérmico}

Sob condições fisiológicas normais, o epitélio gastrintestinal passa por um processo contínuo de proliferação, diferenciação e maturação celular. Os mecanismos precisos que regulam e coordenam essa rápida modificação não são conhecidos, mas o EGF parece desempenhar um papel importante no processo.

O EGF é um peptídeo que produz uma variedade de respostas biológicas - a maioria envolvendo regulação da replicação, movimento e sobrevivência celular. Pertence a uma família de ligantes relacionados (que inclui o fator transformador de crescimento alfa, TGF- $\alpha$ ), que compartilham uma seqüência de aminoácidos homóloga e com alta afinidade pelo mesmo receptor, o EGF-R. As ações biológicas do EGF são mediadas por meio da ligação a este receptor especifico (EGF-R), distribuído pelo trato gastrintestinal fetal e neonatal ${ }^{(1,2,11,13,14,18-22)}$.
Em situações patológicas, o EGF contribui para a proteção epitelial contra agressões e reparo da mucosa pós-lesão. Em contraste, a agressão crônica da mucosa resulta no aparecimento de uma nova linhagem celular, produtora de EGF. Vários estudos de Warner et al demonstraram um papel chave do EGF no processo de cicatrização do trato gastrintestinal, após extensa ressecção do intestino delgado. Administração enteral e parenteral de EGF foi capaz de otimizar a adaptação intestinal, após a ressecção do intestino delgado ${ }^{(1,2,11,13,14,18-22)}$.

Diversos estudos experimentais foram feitos, aperfeiçoando o conhecimento sobre o EGF e seu papel no crescimento e reparo intestinal. Tais estudos sugerem que a administração luminal e sistêmica de EGF poderia ser uma ferramenta poderosa para a proteção celular e estímulo do reparo e regeneração do intestino. Em um modelo animal, a suplementação de EGF em fórmulas baseadas no leite de vaca resultou na redução dramática de $50 \%$ na incidência de $\mathrm{ECN}^{(12,11,13,14,18-22)}$.

Sullivan et al utilizaram EGF em uma criança com necrose intestinal semelhante à enterocolite necrotizante. Tal criança foi tratada com infusão contínua de EGF e, após quatro dias, a estrutura intestinal gravemente danificada foi recoberta e cicatrizada. Em 2007, foi publicado um pequeno trabalho randomizado, com quatro neonatos recebendo placebo e quatro recebendo EGF recombinante humano $100 \mathrm{ng} / \mathrm{kg} / \mathrm{h}$. Os pacientes que receberam EGF mostraram melhora significativa, tanto clínica quanto histológica, do trato gastrintestinal. Entretanto, esse estudo, que tinha previsão para incluir 20 pacientes, foi interrompido após a inclusão do oitavo RN por questões administrativas ${ }^{(23)}$.

\section{Estratégias de prevenção da ECN}

Até o momento não existe base científica suficiente a respeito da eficácia, segurança e custo-benefício que sustente o uso de fatores de crescimento no controle da $\mathrm{ECN}$; portanto, sua administração encontra-se restrita a protocolos de pesquisa e terapias de resgate, em casos extremamente graves. A melhor prática médica disponível, por enquanto, é a prevenção do desenvolvimento da doença, por meio de melhores práticas alimentares, uso de leite humano e fechamento precoce do canal arterial, quando patente.

\section{Práticas alimentares para a prevenir a ECN}

Especialmente após a introdução da nutrição parenteral, a prática de pausa alimentar em RN pré-termo foi popularizada, com base na associação observada entre o início da 
alimentação enteral e o desenvolvimento da ECN. Entretanto, tal prática aumenta o risco de atrofia da mucosa e maior permeabilidade, relatadas em animais ${ }^{(24)}$.

A introdução tardia do alimento parece apenas retardar o início da ECN. Reber $e t$ al ${ }^{(24)}$ revisaram trabalhos sobre este tópico. Alguns deles indicam que, quando são instituídos protocolos alimentares agressivos, a velocidade de progressão da dieta parece contribuir de maneira mais importante para a incidência de ECN. Entretanto, vários outros não foram capazes de demonstrar o mesmo efeito. As questões da rapidez versus lentidão e da precocidade versus atraso na alimentação para prevenir a ECN se mantêm sem resposta ${ }^{(24,25)}$.

Muitos neonatologistas recomendam dieta enteral mínima ou dieta trófica, com o objetivo de estimular a maturação da função gastrintestinal. Entretanto, também não se comprovou a diminuição na incidência de ECN com o uso de tal estratégia ${ }^{(24,25)}$. Berseth et al estudaram dois grupos de neonatos: o primeiro recebeu dieta trófica com $20 \mathrm{~mL} / \mathrm{kg} /$ dia nos primeiros dez dias de vida, com progressão lenta, e o outro, dieta de $20 \mathrm{~mL} / \mathrm{kg} / \mathrm{dia}$ com progressão rápida para $140 \mathrm{~mL} / \mathrm{kg} /$ dia até o décimo dia do estudo. A ECN foi diagnosticada em apenas um dos 71 pacientes do grupo de dieta enteral mínima com progressão lenta e em sete dos 70 recém-nascidos do grupo de progressão rápida da dieta $(p<0,03)^{(26,27)}$. Após este trabalho, tem se recomendado o uso de pequenos volumes de dieta, com progressão lenta do volume diário ofertado ao prematuro.

\section{Corticoterapia antenatal na prevenção da ECN}

O uso antenatal de corticóides para induzir a maturação pulmonar está bem estabelecido. Muitos estudos buscaram estes mesmos efeitos em relação ao trato gastrintestinal, objetivando diminuir a incidência de ECN. Entretanto, os resultados são controversos. Alguns estudos mostraram uma diminuição significativa na incidência de ECN em neonatos cujas mães haviam recebido corticóide antenatal. Por outro lado, outros observaram que a exposição materna ao corticóide antenatal foi associada a risco aumentado para ECN. Apesar disso, a rotina de administração antenatal de corticóide continua sendo a melhor prática médica, objetivando a maturação pulmonar. Novos estudos são necessários para definir mais claramente a associação entre a administração de corticóide e a incidência de $\mathrm{ECN}^{(24)}$.

\section{Imunoglobulinas na prevenção da ECN}

Como RN prematuros possuem níveis diminuídos de imunoglobulinas, particularmente $\operatorname{Ig} \mathrm{A}$ secretora, alguns investigadores avaliaram a administração profilática de imunoglobulina e a incidência de ECN. As evidências disponíveis não dão suporte à administração de imunoglobulina oral para prevenção de ECN. Apesar do uso de imunoglobulinas intravenosas mostrar diminuição na incidência de infecção nosocomial, também não foi demonstrada diminuição da freqüência de ECN em prematuros ${ }^{(24)}$.

\section{Suplementação de aminoácidos na prevenção da ECN}

$\mathrm{O}$ vasodilatador óxido nítrico (NO) tem recebido considerável atenção por se relacionar à regulação do fluxo sanguíneo intestinal, assim como pelo seu papel na manutenção da integridade intestinal. Uma deficiência relativa de arginina leva à inadequada produção de $\mathrm{NO}$, podendo predispor a níveis teciduais inadequados em prematuros, com vasoconstrição, seguida de fenômeno de isquemia e reperfusão, o que propiciaria o desenvolvimento de ECN.

As concentrações de L-arginina no plasma parecem ser significativamente menores em lactentes com ECN. Becker et al mostraram que neonatos com ECN têm deficiência seletiva de aminoácidos, incluindo níveis reduzidos de glutamina aos sete, 14 e 21 dias de vida $(p<0,05)$ e arginina aos sete e 14 dias de vida $(p<0,05)$, comparados a controles sem ECN, o que poderia predispor à doença ${ }^{(28)}$. Em lactentes prematuros, a suplementação de L-arginina foi capaz de reduzir a incidência de ECN. No estudo de Amin et al, a ECN se desenvolveu em cinco dos 75 lactentes do grupo suplementado, em comparação a 21 lactentes dos 77 do grupo controle $(p<0,001)^{(29)}$.

Embora novos estudos sejam necessários para esclarecer o papel dos aminoácidos, como a L-arginina e L-carnitina, na patogênese da ECN, a suplementação da L-arginina é uma grande promessa para a prevenção dessa doença.

\section{Antibióticos orais na prevenção da ECN}

Com base na fisiopatologia da ECN, considerando-se como fator de risco a colonização e subseqüente proliferação bacteriana danificando a superfície da mucosa intestinal, alguns autores têm investigado o uso de antibiótico oral para prevenir a doença. Trabalhos iniciais com aminoglicosídeos tiveram resultados conflitantes, tendo esta prática caído em desuso no final da década de 1970. Em 1998, foi avaliada a eficácia da vancomicina oral na profilaxia da ECN. Dos 71 pacientes do grupo tratado, nove desenvolveram ECN, comparados a 19 dos 69 prematuros do 
grupo controle $(p=0,035)^{30}$. Apesar de, em cinco estudos, a antibioticoterapia oral ter reduzido a incidência de ECN e de óbitos relacionados à ECN em neonatos de muito baixo peso, possíveis efeitos prejudiciais, particularmente o desenvolvimento de resistência bacteriana, fazem com que não seja indicada a administração rotineira de antibióticos orais profiláticos para prevenção de $\mathrm{ECN}^{(24)}$.

\section{Fechamento do canal arterial e prevenção de ECN}

A persistência do canal arterial na vida pós-fetal pode levar à hipoperfusão tecidual, estando implicada no desenvolvimento de ECN. Seu fechamento cirúrgico precoce reduz o risco de ECN. Já o uso de indometacina para o fechamento do canal arterial sintomático pode estar associado à maior incidência de ECN, presumivelmente por diminuição de fluxo sanguíneo tecidual. No entanto, as evidências atuais não associam a indometacina profilática ao risco aumentado de $\mathrm{ECN}^{(24,31)}$.

\section{Prebióticos e probióticos na prevenção da ECN}

Muitos fatores afetam a colonização bacteriana do intestino neonatal, incluindo o uso de antibióticos, o isolamento do paciente, a idade gestacional e o tipo de parto.

Probióticos, como as bifidobactérias, são microorganismos vivos capazes de melhorar o balanço da microbiota intestinal do hospedeiro. Sua eficácia já foi demonstrada na prevenção da ECN, em modelos animais. Em neonatos humanos, Hoyos ${ }^{(32)}$ demonstrou redução na incidência de ECN após alimentação enteral com espécies de Lactobacillus; entretanto, há restrições metodológicas ao estudo, pois o autor usou controles históricos e administrou Lactobacillus para todos os neonatos admitidos na Unidade de Terapia Intensiva.

Os prebióticos, por sua vez, são ingredientes alimentares não digeríveis, como as oligofrutoses, que estimulam de forma seletiva o crescimento e/ou a ativação de espécies bacterianas potencialmente benéficas, presentes no cólon. $\mathrm{O}$ uso da oligofrutose na prevenção da ECN já foi testado em modelo animal $^{(33)}$ e está em estudo em prematuros ${ }^{(34)}$.

Ainda há necessidade de investigação adicional para elucidar o papel dos pré e probióticos na prevenção da ECN, embora pareça promissor.

\section{Conclusões}

Neonatos prematuros não amamentados com leite materno perdem a oportunidade de exposição a peptídeos biologicamente ativos, como o EGF e o G-CSF. Tal fato pode resultar em maior susceptibilidade à lesão intestinal e comprometimento das funções intestinais essenciais.

O uso de fatores de crescimento no tratamento e prevenção da ECN neonatal tem mostrado bons resultados iniciais. Entretanto, novos ensaios clínicos são necessários para verificar a eficácia e a segurança dessas drogas.

Outras medidas, como a suplementação da dieta com arginina e o uso de prebióticos e probióticos para a prevenção da ECN, estão em avaliação. Enquanto isso, a melhor prática médica é a prevenção da doença, com estímulo ao uso do leite materno e progressão lenta da dieta.

\section{Referências bibliográficas}

1. Warner BB, Ryan AL, Seeger K, Leonard AC, Erwin CR, Warner BW. Ontogeny of salivary epidermal growth factor and necrotizing enterocolitis. J Pediatr 2007;150:358-63.

2. Dvorak B, Halpern MD, Holubec H, Williams CS, McWilliam DL, Dominguez JA et al. Epidermal growth factor reduces the development of necrotizing enterocolitis in a neonatal rat model. Am J Physiol Gastrointest Liver Physiol 2002;282:G156-64.

3. Tannuri U. Emergências cirúrgicas abdominais do recém-nascido. Pediatr Mod 2000;36:219-31.

4. de Oliveira ND, Miyoshi MH. Advances in necrotizing enterocolitis. J Pediatr (Rio J) 2005;81( Suppl 1):S16-22.

5. McCormack CJ, Emmens RW, Putnam TC. Evaluation of factors in high risk neonatal necrotizing enterocolitis. J Pediatr Surg 1987;22:488-91.

6. Bell MJ, Ternberg JL, Feigin RD, Keating JP, Marshall R, Barton L et al. Neonatal necrotizing enterocolitis. Therapeutic decisions based upon clinical staging. Ann Surg 1978;87:1-7.

7. Walsh MC, Kliegman RM. Necrotizing enterocolitis: treatment based on staging criteria. Pediatr Clin North Am 1986;33:179-201.
8. Henry MC, Moss RL. Current issues in the management of necrotizing enterocolitis. Semin Perinatol 2004;28:221-33.

9. Dvorak B, Halpern MD, Holubec H, Dvorakova K, Dominguez JA, Williams CS et al. Maternal milk reduces severity of necrotizing enterocolitis and increases intestinal IL-10 in a neonatal rat model. Pediatr Res 2003;53:426-33.

10. Simmonds A, LaGamma EF. Addressing the "New" NEC: Part I: rediscovering the basics. Indian J Pediatr 2006;73:1011-8.

11. Warner BW. NEC, EGF, milk, and spit. Gastroenterology 2002;123:383-4

12. Calhoun DA, Juul SE, McBryde EV, Veerman MW, Christensen RD et al. Stability of filgrastim and epoetin alfa in a system designed for enteral administration in neonates. Ann Pharmacother 2000;34:1257-61.

13. Caplan M. Is EGF the Holy Grail for NEC? J Pediatr 2007;150:329-30.

14. Helmrath MA, Shin CE, Fox JW, Erwin CR, Warner BW. Epidermal growth factor in saliva and serum of infants with necrotising enterocolitis. Lancet 1998;351:266-7.

15. Nowicki PT. Ischemia and necrotizing enterocolitis: where, when, and how Semin Pediatr Surg 2005;14:152-8. 
16. Calhoun DA, Maheshwari A, Christensen RD. Recombinant granulocytecolony-stimulating factor administered enterally to neonates is not absorbed. Pediatrics 2003;112:421-3.

17. Canpolat FE, Yurdakök M, Korkmaz A, Yiğit S, Tekinalp G. Enteral granulocyte colony-stimulating factor for the treatment of mild (stage I) necrotizing enterocolitis: a placebo-controlled pilot study. J Pediatr Surg 2006;41:1134-8.

18. Dvorak B. Epidermal growth factor and necrotizing enterocolitis. Clin Perinatol 2004;31:183-92.

19. Feng J, El-Assal ON, Besner GE. Heparin-binding EGF-like growth factor (HBEGF) and necrotizing enterocolitis. Semin Pediatr Surg 2005;14:167-74.

20. Shin CE, Falcone RA Jr, Stuart L, Erwin CR, Warner BW. Diminished epidermal growth factor levels in infants with necrotizing enterocolitis. J Pediatr Surg 2000;35:173-6.

21. Stern LE, Erwin CR, O'Brien DP, Huang F, Warner BW. Epidermal growth factor is critical for intestinal adaptation following small bowel resection. Microsc Res Tech 2000;51:138-48.

22. Warner BW, Warner BB. Role of epidermal growth factor in the pathogenesis of neonatal necrotizing enterocolitis. Semin Pediatr Surg 2005;14:175-80.

23. Sullivan PB, Lewindon PJ, Cheng C, Lenehan PF, Kuo BS, Haskins JR et al. Intestinal mucosa remodeling by recombinant human epidermal growth factor(1-48) in neonates with severe necrotizing enterocolitis. J Pediatr Surg 2007;42:462-9.

24. Reber KM, Nankervis CA. Necrotizing enterocolitis: preventative strategies. Clin Perinatol 2004;31:157-67.

25. Diehl-Jones WL, Askin DF. Nutritional modulation of neonatal outcomes. AACN Clin Issues 2004;15:83-96.
26. Berseth CL, Bisquera JA, Paje VU. Prolonging small feeding volumes early in life decreases the incidence of necrotizing enterocolitis in very low birth weight infants. Pediatrics 2003;111:529-34.

27. Berseth CL. Feeding strategies and necrotizing enterocolitis. Curr Opin Pediatr 2005;17:170-3.

28. Becker RM, Wu G, Galanko JA, Chen W, Maynor AR, Bose CL et al. Reduced serum amino acid concentrations in infants with necrotizing enterocolitis. J Pediatr 2000;137:785-93.

29. Amin HJ, Zamora SA, McMillan DD, Fick GH, Butzner JD, Parsons HG et al. Arginine supplementation prevents necrotizing enterocolitis in the premature infant. J Pediatr 2002;140:425-31.

30. Siu YK, Ng PC, Fung SC, Lee CH, Wong MY, Fok TF et al. Double blind, randomized, placebo controlled study of oral vancomycin in prevention of neonatal enterocolitis in preterm very low birth weight infants. Arch Dis Child Fetal Neonatal Ed 1998;79:F105-9.

31. Patole SK, Kumaran V, Travadi JN, Brooks JM, Doherty DA. Does patent ductus arteriosus affect feed tolerance in preterm neonates? Arch Dis Child Fetal Neonatal Ed 2007;92:F53-5.

32. Hoyos AB. Reduced incidence of necrotizing enterocolitis associated with enteral administration of Lactobacillus acidophilus and Bifidobacterium infantis to neonates in an intensive care unit. Int J Infect Dis 1999;3:197-202.

33. Butel MJ, Waligora-DuprietAJ, Szylit O. Oligofructose and experimental model of neonatal necrotising enterocolitis. Br J Nutr 2002;87 (Suppl 2):S213-9.

34. Butel MJ, Suau A, Campeotto F, Magne F, Aires J, Ferraris L et al. Conditions of bifidobacterial colonization in preterm infants: a prospective analysis. J Pediatr Gastroenterol Nutr 2007;44:577-82 